\title{
Radiologic Cervical Parameters To Determine Ligamentous Injuries in Patients With Ankylosing Spondylitis - Are They Different?
}

Henrik Teuber ( $\nabla$ henrik.teuber@gmail.com )

Zurich University Hospital https://orcid.org/0000-0002-5022-6644

Sascha Halvachizadeh

University Hospital of Zurich

Melvin Muthirakalayil

Universitat Zurich

Luxu Yin

University Hospital Aachen: Universitatsklinikum Aachen

Harry Eisenkrein

University Hospital Aachen: Universitatsklinikum Aachen

Frank Hildebrand

University Hospital Aachen: Universitatsklinikum Aachen

Philipp Kobbe

University Hospital Aachen: Universitatsklinikum Aachen

Kai Sprengel

Zurich University Hospital

Ladislav Mica

University Hospital Zurich

Hatem Alkadhi

Zurich University Hospital

Hans-Christoph Pape

Zurich University Hospital

Roman Pfeifer

Zurich University Hospital

\section{Research}

Keywords: Ankylosing spondylitis, cervical spine, computed tomography, radiologic parameters

Posted Date: November 2nd, 2021

DOI: https://doi.org/10.21203/rs.3.rs-1025148/v1 
License: (c) (i) This work is licensed under a Creative Commons Attribution 4.0 International License. Read Full License 


\section{Radiologic cervical parameters to determine ligamentous injuries in patients with ankylosing spondylitis - are they different?}

\section{Authors:}

Henrik Teuber $^{1 *}$, Sascha Halvachizadeh ${ }^{1}$, Melvin Muthirakalayil ${ }^{1}$, Luxu Yin ${ }^{2}$, Harry

Eisenkrein $^{2}$, Frank Hildebrand ${ }^{2}$, Philipp Kobbe ${ }^{2}$, Kai Sprengel ${ }^{1}$, Ladislav Mica ${ }^{1}$,

Hatem Alkadhi ${ }^{3}$, Hans-Christoph Pape ${ }^{1}$, Roman Pfeifer ${ }^{1}$

1. Department of Trauma Surgery and Harald-Tscherne Laboratory, University Hospital Zurich, University of Zurich; Zurich, Switzerland.

2. Department of Orthopedics Trauma Surgery, University Hospital Aachen, RWTH Aachen University, Aachen, Germany.

3. Department of Radiology, University Hospital Zurich, University of Zurich; Zurich, Switzerland.

*Corresponding Author

\section{Email addresses:}

Henrik Teuber: henrik.teuber@gmail.com

Sascha Halvachizadeh: sascha.halvachizadeh@usz.ch

Melvin Muthirakalayil: melvin.muthirakalayil@uzh.ch

Luxu Yin:1uxu.yin@,rwth-aachen.de

Harry Eisenkrein: h.eisenkrein@,khporz.de

Frank Hildebrand: fhildebrand@ukaachen.de

Philipp Kobbe: pkobbe@ukaachen.de

Kai Sprengel: kai.sprengel@usz.ch

Ladislav Mica: 1adislav.mica@usz.ch

Hatem Alkadhi: hatem.alkadhi@usz.ch

Hans-Christoph Pape: hans-christoph.pape@usz.ch

Roman Pfeifer: roman.pfeifer@usz.ch 


\section{Abstract}

Introduction: Cervical ligamentous injuries in patients with ankylosing spondylitis (AS) may be difficult to detect, even with the utilization of computed tomography (CT) scans. The purpose of this study was to investigate the influence AS has on various radiologic parameters used to detect traumatic and degenerative pathologies of the cervical spine.

Methods: A matched, case-control retrospective analysis of patients with AS and controls without AS admitted at two level-1 trauma centers was performed. All patients were admitted via shock room and received a polytrauma CT. Study patients were included if they had no injury to the cervical spine. Twenty-four CT parameters of atlanto-occipital dislocation/ instability, traumatic and degenerative spondylolisthesis, basilar invagination, and prevertebral soft-tissue swelling were assessed. Study patients were matched by age and sex.

Results: A total of 78 patients were included (AS group, $n=39$; control group, $n=39$ ). The evaluated cervical radiologic parameters were largely within normal limits and showed no significant clinical or morphologic differences between the two groups.

Conclusion: In this analysis, CT measurements pertaining to various cervical pathologies were not different between patients with and without ankylosing spondylitis. Parameters to assess for atlanto-occipital dislocation/ instability, spondylolisthesis, or basilar invagination may reliably be used in patients with AS.

Keywords: Ankylosing spondylitis, cervical spine, computed tomography, radiologic parameters 


\section{Declarations}

\section{Ethics approval and consent to participate}

The study was approved by the respective local ethics committees of the two level 1 trauma centers.

\section{Consent for publication}

The patient who`s CT image is shown in Figure 1 has a signed general consent form.

\section{Availability of data and materials}

The data generated and analysed for the current study are not publicly available, but are available from the corresponding author on reasonable request.

\section{Competing interests}

The authors declare that they have no competing interests.

\section{Funding}

No external funding was necessary in completing this retrospective study.

\section{Authors' contributions}

FH, PK, KS, LM, HP, and RP have made substantial contributions to the conception and design of the study. HT, SH, MM, LY, HE, HP and RP have made substantial contributions in the acquisition, analysis, and interpretation of data. HT, SH, MM, LY, HE, HA, LM, HP and RP have drafted the work or substantively revised it. All authors read and approved the final manuscript.

\section{Acknowledgements}

None 


\section{Introduction}

Ankylosing Spondylitis (AS) is a progressive arthritis that primarily affects the axial skeleton, which often results in multi-segmental fusion of the spine. The loss of mobility in the vertebral column results in a rigid structure that behaves like a long bone. In association with osteoporosis often accompanying AS in the elderly, these individuals are significantly more susceptible to unstable fractures of the spine. Cervical spine involvement, especially in late-stage disease, is common in patients with AS. Further, patients with early AS exhibit cervical-predominant pathology in $5.2 \%$ of cases. In patients with AS for at least 20 years, $19.9 \%$ of males and $16.0 \%$ of females exhibited cervical-predominant pathology [1]. After 25 years of disease, 75\% of AS patients had cervical spine involvement, while $85 \%$ had lumbar spine involvement [2].

Multiple studies have shown progressive pathologic changes to the cervical spine in patients with longer disease duration [3]. Specifically, physiologic cervical lordosis may regress and pathologic cervical kyphosis may develop in AS patients, which is the result of spondylarthropathy. Patients unload the inflamed facet joints by assuming a kyphotic posture. Ultimately with increasing syndesmophyte formation and eventual ankylosis, the cervical spine assumes a fixed kyphotic deformity $[4,5]$. Cervical involvement in AS patients requires particular attention, as the risk of fractures and severe neurological complications due to spinal stenosis is greatly elevated $[3,6]$.

However, little is known about the effect AS has on characteristic radiologic changes found in common traumatic or degenerative pathologies of the cervical spine. We hypothesized that the ankylosis, spinal degeneration and deformity found in AS may affect important radiologic parameters used in detecting potentially subtle traumatic or degenerative cervical instability including atlanto-occipital dislocation, spondylolisthesis, and basilar invagination. To assess for potential morphologic changes of the cervical spine due to AS, an analysis of 24 commonly used radiologic 
parameters were measured in cervical computed tomographys (CTs) of non-injured patients with AS and non-injured controls without AS.

\section{Patients and methods}

The study was approved by the respective local ethics committees of the two level 1 trauma centers. All patients were treated at two level 1 trauma centers between 2009 and 2019. All patients were admitted via shock room and received a polytrauma CT. Patients may have had multiple injuries, but were included only if they had no injuries to the cervical spine. In the study group, patients with a confirmed diagnosis of AS (fulfilment of the modified New York criteria for AS or ASAS criteria for axial SPA) were included. Study group patients were matched 1:1 for age and gender with patients without AS in whom cervical spinal injuries were ruled out.

Cervical spine CT examinations: All patients underwent scans by multi-detector row CT of the cervical spine. Axial images were reconstructed at a section thickness of $1 \mathrm{~mm}$ and an increment of $0.7 \mathrm{~mm}$. Axial, coronal, and sagittal reformations were obtained. Twenty-four radiologic parameters summarized in Table 1 were measured and evaluated by three independent orthopedic surgeons who were blinded to clinical data. An example of a sagittal plane CT of the cervical spine of a patient in the AS group is shown in Figure 1.

Statistics: Data were analyzed using the SPSS software package version 20.0 for Windows (SPSS Inc., Chicago, IL, USA). Descriptive statistics are presented as mean and standard error of the mean (SEM). Statistical distribution of all the data was assessed by both the Shapiro-Wilk and Kolmogorov-Smirnov tests. An independent ttest was used to calculate statistical differences between the AS group and the control group. A p-value less than 0.05 was considered statistically significant. 


\section{Results}

Overall, 78 patients were included in the study. The AS study group consisted of 35 male and 4 female patients with an average age of 66.2 years and was matched on a 1:1 basis with healthy controls. AS patients had a mean New York criteria sacroilitis grade of 2.89 (SD 0.76). Both groups were demographically very similar. However, steroid use was unsurprisingly significantly higher in the AS vs. control study group (10.3\% vs. $0.0 \%$ of patients, $p=0.044)$. Study demographics are shown in Table 2 .

Overall, the radiologic parameters measured were within normal limits and showed little variation between the groups. None of the 24 CT-based parameters measured were statistically or clinically different between the groups and are summarized in Table 3.

\section{Atlanto-occipital dislocation/ instability parameters:}

The BDI and BAI were both well within normal limits $(<8.5 \mathrm{~mm})$ and similar in both the AS and control groups ( 5.1 vs. $5.3 \mathrm{~mm}, \mathrm{p}=0.544$ and 4.9 vs. $5.6 \mathrm{~mm}, \mathrm{p}=0.180$, respectively). Both methods of measuring Powers Ratio were also similar and within normal limits $(<0.9)$ in the AS and control groups ( 0.87 vs $0.90, p=0.080$ and 0.74 vs $0.78, p=0.059$, respectively). Eleven patients showed pathological Lee X-Line parameters in both study groups $(28.2 \%, \mathrm{p}=1.00)$. Further, we looked at the lengths of the individual lines used to assess Powers Ratio and Lee X-Lines as well as measuring a Lee X-Line ratio to see if we could find any subtle anatomic variation between the two groups. This analysis however also showed no differences between the groups. The mean posterior atlanto-dens-interval (PADI) was well within normal limits $(>14 \mathrm{~mm})$ and nearly identical in the AS and control groups $(21.0 \mathrm{vs} .21 .3 \mathrm{~mm}$, $\mathrm{p}=0.523)$.

\section{Basilar invagination parameters:}

Both Chamberlain's and McCrae's lines showed similar results in both the AS group and healthy controls. Seven patients in the control group and 8 patients in the AS 
group had a pathological Chamberlain line (17.9\% vs. $20.5 \%$ respectively, $\mathrm{p}=1.00)$. There were no patients with a pathological McCrae's line in either group $(0 \%$, $\mathrm{p}=1.00)$. As with the atlanto-occipital parameters above, line lengths were also compared to observe potentially subtle anatomic differences, but no differences between the groups were observed.

\section{Degenerative/traumatic spondylolisthesis parameters:}

Endplate tilt at $\mathrm{C} 2 / 3\left(1.8^{\circ}\right.$ vs $\left.1.9^{\circ}, \mathrm{p}=0.910\right), \mathrm{C} 4 / 5\left(1.6^{\circ}\right.$ vs $\left.-1.4^{\circ}, \mathrm{p}=0.082\right)$ and $\mathrm{C} 5 / 6$ $\left(2.6^{\circ}\right.$ vs $\left.1.8^{\circ}, \mathrm{p}=0.597\right)$ were similar and stable $\left(<11^{\circ}\right)$ between the AS and control groups. However, a consistent, but subtle trend toward more kyphotic angulation, especially at the C4/5 level, was seen in the AS group. Displacement at the level of $\mathrm{C} 3$ and $\mathrm{C} 6$ were less than $1 \mathrm{~mm}$ in both the AS and control patients. Finally, soft tissue swelling at C3 (4.6 vs $6.1 \mathrm{~mm}, \mathrm{p}=0.070)$ and C6 (13.8 vs $12.4 \mathrm{~mm}, \mathrm{p}=0.107)$ were within normal limits $(\mathrm{C} 3<7 \mathrm{~mm}, \mathrm{C} 6<21 \mathrm{~mm})$ and similar in both the AS and control groups, respectively. 


\section{Discussion}

In this study, despite known morphologic changes to the cervical spine in the setting of AS, none of the cervical radiologic parameters measured in patients with AS were different compared to healthy controls. We could therefore not support the study hypothesis that AS may affect normal values of commonly measured radiologic parameters used in diagnosing atlanto-occipital dislocation, cervical spondylolisthesis and basilar invagination.

Cervical trauma in patients with AS is associated with a high risk of potentially severe neurologic complications [7]. Early awareness and recognition of cervical injuries is, therefore especially important in the setting of AS. However, the assessment of cervical instability or fractures with conventional imaging in patients with AS is challenging. Conventional plain radiographs show low efficiency in diagnosing cervical injuries due to diffuse ossification of cervical spinal ligaments, joints and discs [8]. In a retrospective review, Anwar et al., showed that $60 \%$ of cervical fracture dislocations in patients with AS were undetectable in initial radiographs [9]. Sensitivity for detecting cervical injuries is much higher in CT with an improved sensitivity of up to $98 \%$ [10-12]. Thus, the indication for conventional radiologic imaging of the cervical spine is limited and CT of the cervical spine has become the standard imaging modality when cervical trauma is suspected $[13,14]$. This is especially true in the setting of AS.

\section{Parameters assessing atlanto-occipital stability are unaltered in AS patients}

BAI and BDI are helpful for the diagnosis of atlanto-occipital dissociation injuries. Normal values should be less than $12 \mathrm{~mm}$ on plain radiographs. An increase in this distance may indicate instability [15]. Multiple studies declared that the accepted ranges of normal values of BDI and BAI on plain radiographs cannot apply to CT images. Rojas et al. [16] argued that the BAI was difficult to reproduce on CT images; the value was found to be highly variable and a number of subjects had BAIs greater than $12 \mathrm{~mm}$. They found that the distance of BDI was $<8.5 \mathrm{~mm}$ in the vast 
majority of 200 cases and the maximum distance recorded was $9.1 \mathrm{~mm}$. Gonzalez et al. [17] also demonstrated a mean BDI of $4.7 \mathrm{~mm}$ and a maximum of $9 \mathrm{~mm}$ in CT images from healthy individuals. In line with the literature, the BDI and BAI in the control group of our study were well within normal limits at 5.3 (SD 1.6) and 5.6 (SD 2.2) $\mathrm{mm}$, respectively. Neither BDI nor BAI differed in the AS group compared to the control group and were equally well within normal limits.

Changes to the craniovertebral junction in AS with cervical involvement has been well described and is frequently involved in severe AS [18]. All patients with AS underwent structural changes of articulation or ligamentous structures [18]. The interval of the atlanto-occipital joint and the atlanto-dental joint were decreased in AS patients, but the BDI and the Power's ratio were not changed [18]. Our results confirm these findings.

Studies of BAI measurements in AS patients are rare. Robust articular ligaments, such as the cruciform ligament, are important for atlanto-axial joint stability [19]. In the pathological process of AS, these ligaments undergo inflammation such as enthesitis, and laxity or rupture with subsequent atlanto-axial subluxation [20]. Furthermore, odontoid pannus formation can lead to atlanto-axial instability [21]. We suspected that excessive kyphosis might also occur at this level, potentially altering various parameters, especially BAI. However, BAI was found within normal limits and not different to the control group. The only pathological values we found in the present study were seen with the Lee's X-line parameters with pathological findings in $11(28.2 \%)$ patients in both study arms. The hypothesis of altered atlanto-occipital cervical parameters on account of AS induced instability, specifically pathologic kyphosis, could not be supported in the present study.

\section{Parameters assessing basilar invagination and cervical spondylolisthesis remain stable in AS patients}

The endplate and the posterior vertebral body tangent measurement methods for C2-C3 angulation were first described by Levine and Edwards [22]. C2-C3 angulation and translation were usually used to evaluate for traumatic spondylolisthesis including 
Hangman's fractures [23, 24]. The physiological cervical spine shows a slight lordotic curvature at the level C2-C3 of about $-1.9 \pm 5.20^{\circ}$ [25]. Cervical lordosis in AS patients is decreased and with increasing severity, even cervical kyphosis deformities may occur [4]. However, it is unknown if kyphotic changes in AS are focussed at specific levels or if the kyphotic changes are equally distributed throughout the cervical spine and thus difficult to detect at individual levels. In this study, the angulation at $\mathrm{C} 2-\mathrm{C} 3$ as well as $\mathrm{C} 4-\mathrm{C} 5$ and $\mathrm{C} 5-\mathrm{C} 6$ were measured to assess for potential underlying spondylolisthetic changes [26]. Any bony pathologic changes of the endplate may influence the measurement of these angles. This is especially true in AS patients, where the endplate border is altered by the presence of syndesmophytes characteristic of the disease $[27,28]$. In this study, no changes in single-level angulation were found between the groups. However, a subtle but statistically insignificant trend toward more kyphosis at all levels measured was seen in the AS versus control group. This was most pronounced at the $\mathrm{C} 4 / 5$ level.

Further, results did not differ regardless if measured using the endplate method or the posterior vertebral body line method [26]. We do, however, feel that the posterior vertebral line method may prove more accurate and useful in evaluating the cervical spine in AS, as it measures the angle of lines drawn perpendicular to the posterior vertebral body aspect of the two endplates. The pathological changes of the vertebral edges therefore have less impact on accurate assessment of possible spondylolisthesis. The results suggest that endplate angulation at single vertebral levels remains largely unchanged, despite overall loss of cervical lordosis in AS patients.

Additionally, no differences or pathological values in vertebral displacement (defined as $>3 \mathrm{~mm}$ ) measured at C4-C5 and C5-C6 were found between the groups, further suggesting that spondylolisthetic degeneration was not present in the AS study group.

Parameters assessing basilar invagination were also identical between the AS and control group. While approximately $20 \%$ of patients in both the AS $(n=7)$ and control $(n=8)$ groups had a pathological Chamberlain line, none of the patients in both the AS and control group had a pathological McCrae line. While of no clinical or diagnostic 
significance, the line lengths were also measured as with the above parameters used to assess for atlanto-occipital dissociation. Here as well, we saw no differences between the groups, suggesting that subtle morphologic changes in the AS group were not present. While common in other arthopathies, such as rheumatoid arthritis, basilar invagination is rare in AS as pannus formation and degenerative destruction of the craniocervical junction has only been documented in late stage disease [20, 21, 29]. Our results are therefore in line with the literature and previous radiologic studies of AS.

It is important to note that with increasing severity of AS, more osteophytic and syndesmophytic changes occur, which may have influenced the accuracy of some of the measured parameters that rely on precise bony landmarks. The severity of AS was not specifically assessed for in this study and is an important study limitation, especially considering that cervical changes are usually found in late stage disease. Since the majority of the AS patients in this study population were older, however, it is likely that a significant portion of the study population was afflicted with more severe disease. Due to the high patient age, the matched study design was useful in accounting for the potentially confounding effect of general spinal degeneration.

\section{Conclusion}

Our analysis showed that while cervical changes can be significant in AS, commonly assessed traumatic and degenerative radiologic cervical spine parameters were not different in patients with AS compared to matched controls without AS. This affirms the broad applicability of these radiologic parameters and suggests that their normal values may be used with confidence in patients with AS. 


\section{References}

1. Jang, J.H., et al., Ankylosing spondylitis: patterns of radiographic involvement--a reexamination of accepted principles in a cohort of 769 patients. Radiology, 2011. 258(1): p. 1928.

2. Brophy, S., et al., The natural history of ankylosing spondylitis as defined by radiological progression. J Rheumatol, 2002. 29(6): p. 1236-43.

3. Lee, J.Y., et al., Cervical spine involvement in longstanding ankylosing spondylitis. Clin Exp Rheumatol, 2005. 23(3): p. 331-8.

4. Hoh, D.J., P. Khoueir, and M.Y. Wang, Management of cervical deformity in ankylosing spondylitis. Neurosurg Focus, 2008. 24(1): p. E9.

5. Lazennec, J.Y., H. d'Astorg, and M.A. Rousseau, Cervical spine surgery in ankylosing spondylitis: Review and current concept. Orthop Traumatol Surg Res, 2015. 101(4): p. 507-13.

6. Robinson, Y., B. Sanden, and C. Olerud, Increased occurrence of spinal fractures related to ankylosing spondylitis: a prospective 22-year cohort study in 17,764 patients from a national registry in Sweden. Patient Saf Surg, 2013. 7(1): p. 2.

7. Chaudhary, S.B., H. Hullinger, and M.J. Vives, Management of acute spinal fractures in ankylosing spondylitis. ISRN Rheumatol, 2011. 2011: p. 150484.

8. van Tubergen, A., et al., Are syndesmophytes most prevalent in the lumbar or in the cervical spine in patients with ankylosing spondylitis and do they develop in a specific direction? Rheumatology (Oxford), 2012. 51(8): p. 1432-9.

9. Anwar, F., et al., Delayed presentation and diagnosis of cervical spine injuries in long-standing ankylosing spondylitis. Eur Spine J, 2011. 20(3): p. 403-7.

10. Wintermark, M., et al., Thoracolumbar spine fractures in patients who have sustained severe trauma: depiction with multi-detector row CT. Radiology, 2003. 227(3): p. 681-9.

11. Gale, S.C., et al., The inefficiency of plain radiography to evaluate the cervical spine after blunt trauma. J Trauma, 2005. 59(5): p. 1121-5.

12. Holmes, J.F. and R. Akkinepalli, Computed tomography versus plain radiography to screen for cervical spine injury: a meta-analysis. J Trauma, 2005. 58(5): p. 902-5.

13. Blackmore, C.C., et al., Cervical spine screening with CT in trauma patients: a cost-effectiveness analysis. 1999. 212(1): p. 117-125.

14. Bailitz, J., et al., CT should replace three-view radiographs as the initial screening test in patients at high, moderate, and low risk for blunt cervical spine injury: a prospective comparison. 2009. 66(6): p. 1605-1609.

15. Harris, J.H., Jr., G.C. Carson, and L.K. Wagner, Radiologic diagnosis of traumatic occipitovertebral dissociation: 1. Normal occipitovertebral relationships on lateral radiographs of supine subjects. AJR Am J Roentgenol, 1994. 162(4): p. 881-6.

16. Rojas, C.A., et al., Reassessment of the craniocervical junction: normal values on CT. AJNR Am J Neuroradiol, 2007. 28(9): p. 1819-23.

17. Gonzalez, L.F., et al., Vertical atlantoaxial distraction injuries: radiological criteria and clinical implications. J Neurosurg Spine, 2004. 1(3): p. 273-80.

18. Slobodin, G., et al., Craniocervical junction involvement in ankylosing spondylitis. Eur Spine J, 
2015. 24(12): p. 2986-90.

19. Hall, G.C., et al., Atlanto-occipital dislocation. World J Orthop, 2015. 6(2): p. 236-43.

20. Tam, L.S., J. Gu, and D. Yu, Pathogenesis of ankylosing spondylitis. Nat Rev Rheumatol, 2010. 6(7): p. 399-405.

21. Rajak, R., et al., Odontoid pannus formation in a patient with ankylosing spondylitis causing atlanto-axial instability. BMJ Case Rep, 2012. 2012.

22. Levine, A.M. and C.C. Edwards, The management of traumatic spondylolisthesis of the axis. J Bone Joint Surg Am, 1985. 67(2): p. 217-26.

23. Muthukumar, N., C1-C3 lateral mass fusion for type Ila and type III Hangman's fracture. J Craniovertebr Junction Spine, 2012. 3(2): p. 62-6.

24. Park, J.H., S.H. Kim, and K.H. Cho, Clinical Outcomes of Posterior C2-C3 Fixation for Unstable Hangman's Fracture Compared with Posterior C1-C3 Fusion. Korean J Spine, 2014. 11(2): p. 338.

25. Scheer, J.K., et al., Cervical spine alignment, sagittal deformity, and clinical implications: a review. J Neurosurg Spine, 2013. 19(2): p. 141-59.

26. Bono, C.M., et al., Measurement techniques for upper cervical spine injuries: consensus statement of the Spine Trauma Study Group. Spine (Phila Pa 1976), 2007. 32(5): p. 593-600.

27. Hermann, K.G., et al., Spinal changes in patients with spondyloarthritis: comparison of MR imaging and radiologic appearances. Radiographics, 2005. 25(3): p. 559-69; discussion 569-70.

28. Jurik, A.G., Imaging the spine in arthritis-a pictorial review. Insights Imaging, 2011. 2(2): p. 177191.

29. Pang, D. and D.N.J.C.s.N.S. Thompson, Embryology and bony malformations of the craniovertebral junction. 2011. 27(4): p. 523-564. 
Table 1:

Brief description and method of measurement of the selected radiologic parameters

BA

The distance between the basion and the tangent of the

posterior border of the axis.

The distance between the basion and the tip of the odontoid

BD

C2 - C3 angle

(End-Plate Method)

C2-C3 angle

(posterior method)

Lee X-Line

Power ratio 1

Power ratio 2

Chamberlain line

McCrae's line

PADI

Soft-tissue shadow of C3 and C6

Endplate tilt of $\mathrm{C} 4-\mathrm{C} 5$ and $\mathrm{C5}-\mathrm{C} 6$

Displacement of C4-C5 and C5-C6 process.

Angle between lines tangent to the inferior endplate of $\mathrm{C} 2$

and C3.

Angle between lines tangent to the posterior vertebral body of $\mathrm{C} 2$ and $\mathrm{C} 3$.

Lines of basion to the arch of $\mathrm{C} 2$ and ophistion to the

postero-inferior edge of $\mathrm{C} 2$. When both lines do not intersect

$\mathrm{C} 2$ and $\mathrm{C} 1$ respectively, dissociation is suspected.

The ratio of Basis-arc C1 / Dens-opisthion. A ratio greater

than 0.9 is considered pathological and suggestive of atlantooccipital instability.

The ratio of Basis-Arc C1 / C1 ventral-opisthion.

The line connects the posterior end of the hard palate and the posterior lip of the foramen magnum.

The line of the opening of the foramen magnum

The posterior interval between atlas and dens.

The distance between the anterior aspect of the vertebral

body baseplate to the trachea

Angle of lines drawn along the inferior endplate of $\mathrm{C} 4-\mathrm{C} 5$ and C5-C6.

The displacement of the posteroinferior edge of the superior vertebral body to the posterosuperior edge of the inferior vertebral body. 


\begin{tabular}{lccc}
\hline Table 2: Demographics & & & \\
\hline & Control & $\begin{array}{c}\text { Ankylosing } \\
\text { spondylitis }\end{array}$ & $\mathrm{p}$ \\
\cline { 2 - 4 } $\mathrm{n}$ & 39 & 39 & \\
Age (yrs, mean (SD)) & $66.2(14.4)$ & $66.4(14.2)$ & matched \\
Female (n, \%) & $4(10.3)$ & $4(10.3)$ & matched \\
Height (cm, mean (SD)) & $174.5(6.0)$ & $172.4(4.7)$ & 0.169 \\
Weight (kg, mean (SD)) & $80.7(11.1)$ & $77.4(13.8)$ & 0.334 \\
BMI (mean (SD)) & $26.5(3.1)$ & $26.2(4.5)$ & 0.522 \\
Diabetes Mellitus (n, \%) & $9(23.1)$ & $12(30.8)$ & 0.450 \\
Corticosteroid Therapy (n, \%) & $0(0.0)$ & $4(10.3)$ & 0.044 \\
Osteoporosis (n, \%) & $2(5.1)$ & $3(7.7)$ & 0.649
\end{tabular}


Figure 1: sagittal plane CT of the cervical spine of a patient in the AS group, an 80-year old male

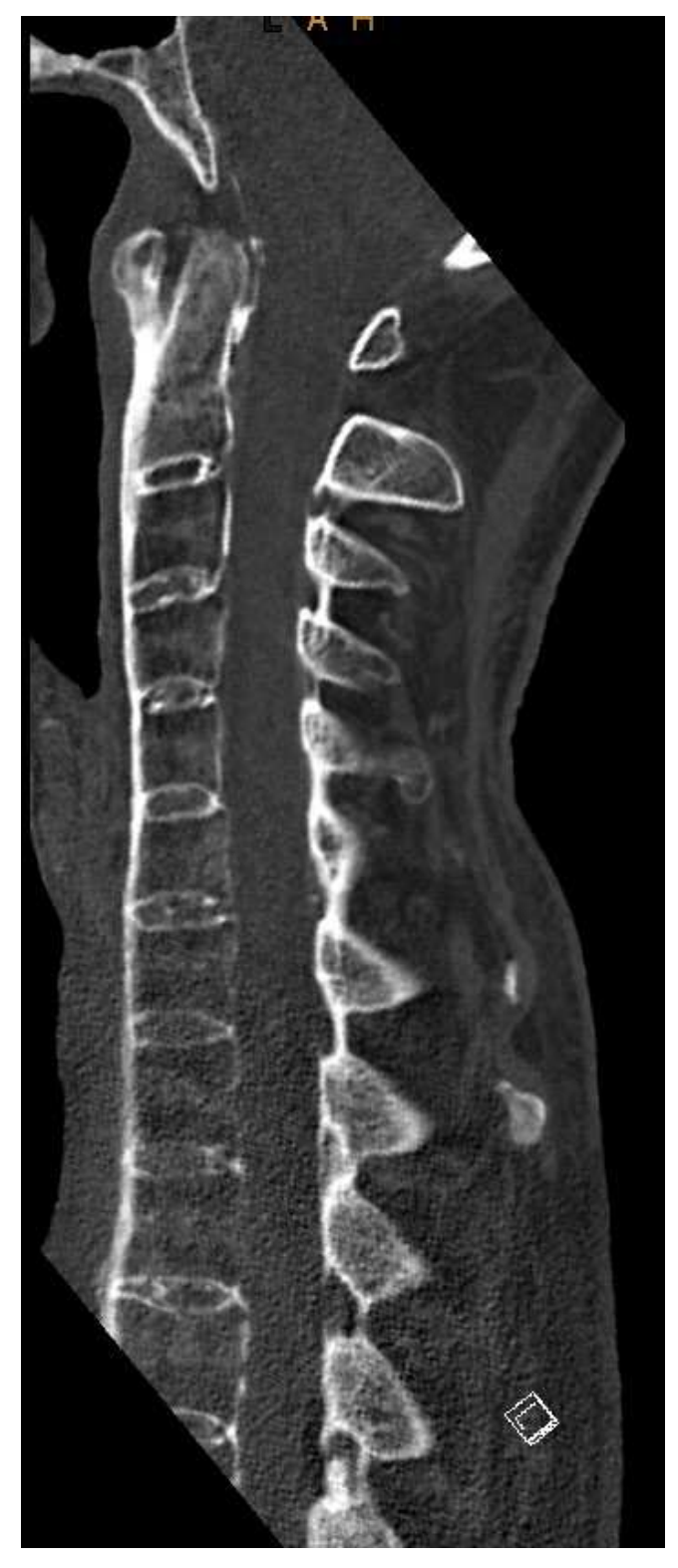


Table 3: Radiologic parameters of various ligamentous injuries

\begin{tabular}{|c|c|c|c|}
\hline & Control & $\begin{array}{l}\text { Ankylosing } \\
\text { Spondylitis }\end{array}$ & $\begin{array}{c}\mathrm{P}- \\
\text { value }\end{array}$ \\
\hline $\mathrm{n}$ & 39 & 39 & \\
\hline $\mathrm{BDI}(\mathrm{mm}$, mean $(\mathrm{SD}))$ & $5.3(1.6)$ & $5.1(2.0)$ & 0.544 \\
\hline BAI (mm, mean (SD)) & $5.6(2.2)$ & $4.9(2.4)$ & 0.180 \\
\hline Basis-C1 (mm, mean (SD)) & $32.8(2.4)$ & $31.7(3.0)$ & 0.092 \\
\hline Dens-Opisthion (mm, mean (SD)) & $36.4(3.0)$ & $36.7(3.1)$ & 0.692 \\
\hline Powers Ratio 1 (mean (SD)) & $0.90(0.08)$ & $0.87(0.10)$ & 0.080 \\
\hline C1-Opisthion (mm, mean (SD)) & $42.5(3.5)$ & $42.8(3.4)$ & 0.690 \\
\hline Powers Ratio 2 (mean (SD)) & $0.78(0.07)$ & $0.74(0.07)$ & 0.059 \\
\hline Basis-C2 (mm) & $42.05(3.32)$ & $40.76(3.39)$ & 0.092 \\
\hline C2-Ophistion (mm) & $44.49(5.96)$ & $43.90(5.34)$ & 0.644 \\
\hline Lee X-line Ratio & $0.96(0.17)$ & $0.94(0.13)$ & 0.484 \\
\hline Pathological X-line (n (\%)) & $11(28.2)$ & $11(28.2)$ & 1.000 \\
\hline Chamberlain line length (mean (SD)) & $82.07(3.99)$ & $81.58(3.75)$ & 0.591 \\
\hline Pathological Chamberlain line (n (\%)) & $7(17.9)$ & $8(20.5)$ & 1.000 \\
\hline McCrae's line length (mean (SD)) & $37.60(2.56)$ & $37.37(3.57)$ & 0.747 \\
\hline Pathological McCrae's line ( $(\%)$ ) & $0(0.0)$ & $0(0.0)$ & 1.000 \\
\hline PADI (mm, mean (SD)) & $21.3(2.3)$ & $21.0(2.1)$ & 0.523 \\
\hline C2-C3 Angulation (end plate) $\left({ }^{\circ}\right.$, mean (SD)) & $1.9(5.2)$ & $1.8(5.6)$ & 0.910 \\
\hline $\mathrm{C} 2-\mathrm{C} 3$ Angulation (posterior wall) $\left({ }^{\circ}\right.$, mean (SD)) & $6.3(6.6)$ & $8.0(7.0)$ & 0.272 \\
\hline End plate tilt C4-C5 $\left({ }^{\circ}\right.$, mean (SD)) & $-1.4(5.9)$ & $1.6(8.6)$ & 0.082 \\
\hline End plate tilt $\mathrm{C} 5-\mathrm{C} 6\left(^{\circ}\right.$, mean (SD)) & $1.8(5.6)$ & $2.6(7.4)$ & 0.597 \\
\hline Displacement C4-C5 (mm, mean (SD)) & $0.6(1.1)$ & $0.4(1.2)$ & 0.642 \\
\hline Displacement C5-C6 (mm, mean (SD)) & $0.3(0.9)$ & $0.4(1.0)$ & 0.651 \\
\hline Soft-tissue shadow C3 (mm, mean (SD)) & $6.1(4.3)$ & $4.6(2.6)$ & 0.070 \\
\hline Soft-tissue shadow C6 (mm, mean (SD)) & $12.4(4.0)$ & $13.8(3.5)$ & 0.107 \\
\hline
\end{tabular}

